\title{
Research Paper \\ A Comparative Study on the Effects of Different Informing Methods on Parental Acceptance of Pediatric Behavior Management Techniques
}

\author{
Yalda Mirmoeini ${ }^{1}\left(\mathbb{D},{ }^{*}\right.$ Hamid Sarlak $^{1}$ (1), Shima Normohammadi ${ }^{1}$ (1), Afrooz Nakhostin ${ }^{1}$ (1), Zohreh Salimi ${ }^{1}$ (1)
}

1. Department of Pediatric Dentistry, Faculty of Dentistry, Arak University of Medical Sciences, Arak, Iran.

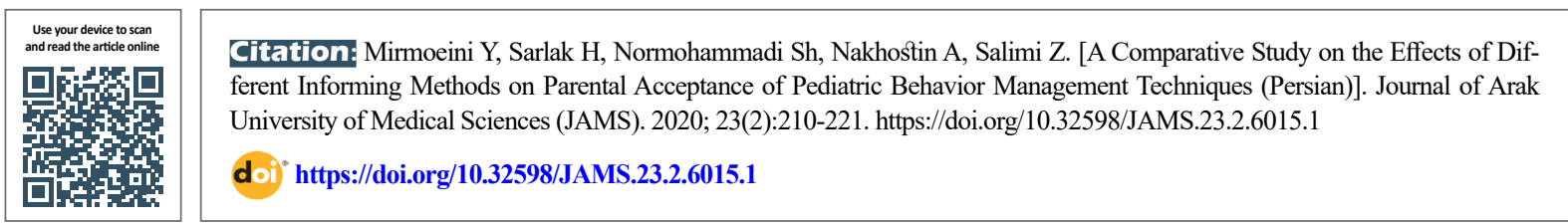

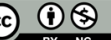

Article Info:

Received: 23 Oct 2019

Accepted: 02 Feb 2020

Available Online: 01 Jun 2020

Key words:

Parental acceptance,

Pediatric dentistry,

Behavior manage-

ment, Informing

\section{A B STRACT}

Background and Aim Parental acceptance is an important parameter in selecting the proper behavior management technique during pediatric dental treatment. The present study aimed to compare the effects of different informing methods on acceptance of parents about behavior management techniques used in pediatric dentistry.

Methods \& Materials This quasi-experimental study was conducted on one 162 parents of 3-7-year-old uncooperative children who were candidates for dental treatment. They were randomly divided into three groups. Their acceptance about six behavior management techniques including general anesthesia, voice control, hand-over-mouth, parental separation, active and passive physical restraint were assessed by a questionnaire. After determining the level of acceptance, the information about the techniques was presented through three ways include writing, verbal and visual methods. Finally, their acceptance of each technique and the reasons for non-acceptance were re-evaluated. Data were analyzed in SPSS v.23 software using ANOVA and t-test.

Ethical Considerations This study obtained its ethical approval from the Research Ethics Committee of Arak University of Medical Sciences under code: IR.ARAKMU.REC.1398.102.

Results The informing methods significantly increased the parents' acceptance about behavior management techniques. According to the ANOVA results, the effect of visual presentation method in general anesthesia technique was significantly lower than that of other methods $(P<0.05)$, while its effect in active physical restraint technique was significantly greater than that of other methods $(P<0.05)$. There was no statistically significant difference between the effects of informing methods in other behavior management techniques.

Conclusion Informing parents about pediatric behavior management techniques increase their acceptance. Informing by visual presentation is the most effective method for increasing their acceptance of physical restraint technique, while in their acceptance of general anesthesia technique other informing methods are more effective

\section{Extended Abstract}

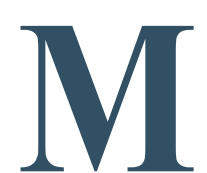

\section{Introduction}

ost children who see a dentist are anxious, and it is common for children not to cooperate during dental treatment [1]. Pediatric dentistry tries to have a positive effect on children. For which purpose, advanced pharmacological and behavior management techniques are used [2, 3]. However, parents may decide not to accept any of these methods [3]. The more positive the attitude of parents towards these methods, the

\section{* Corresponding Author}

Hamid Sarlak, PhD.

Address: Department of Pediatric Dentistry, Faculty of Dentistry, Arak University of Medical Sciences, Arak, Iran

Tel: +98 (936) 4321836

E-mail: dr.hamidsarlak@yahoo.com 
less stress is transferred from the parents to the child and the cooperation of parents with the dentist in using these methods increases [5]. The purpose of informing is to introduce the pediatric behavior management techniques to parents, which may increase their acceptance and satisfaction with these methods. Various methods of providing information to parents include oral/visual presentation and written report [3]. The aim of this study was to compare the effect of different methods of parental informing on their acceptance of behavior management techniques and determine the reasons for their non-acceptance.

\section{Materials and Methods}

This quasi-experimental study was conducted on one 162 parents of 3-7-year-old uncooperative children who were candidates for dental treatment. They were randomly divided into three groups. Their acceptance about 6 behavior management techniques including general anesthesia, voice control, hand-over-mouth, parental separation, active/passive physical restraint were assessed by questionnaire. After determining the level of acceptance, the information was presented via writing report, and oral and visual (film presentation) methods. In written method, the information on each of the behavior management techniques was provided in writing. In visual method, a researcher-made film was produced to demonstrate behavior management techniques. Their acceptance and the reasons for non-acceptance were re-evaluated. Results were analyzed in SPSS V. 23 software using ANOVA and t-test (Table 1 \& Table 2).

\section{Results}

According to participants, the order of acceptance of pediatric behavior management techniques was: parental separation, voice control, active physical restraint, general anesthesia, passive physical restraint, and hand-over-mouth techniques. The effectiveness of all informing methods on increasing the acceptance of pediatric behavior manage- ment techniques by parents was statistically significant. The effect of visual presentation method in general anesthesia technique was lower than that of other methods, while in active physical restraint technique its effect was more than that of other methods. There was no statistical difference in other behavior management techniques, and the effectiveness of all three informing methods was similar. Without categorizing behavior management techniques, the effectiveness of the written informing method was more than the visual informing method and the effect of visual method was more than the oral informing method, but the difference was not statistically significant. The most important reasons for not accepting different pediatric behavior management techniques were as follows:

- General anesthesia technique: Complications and harm to the child;

- Voice control technique: Emotional problems and harm to the child

- Parental separation technique: Lack of positive effect on the child's cooperation, creating emotional problems, worrying about its harm to the child, and believing in a more appropriate and convenient alternative method;

- Hand-over-mouth and active physical restraint techniques: Emotional problems and harm to the child;

- Passive physical restraint: Emotional problems and harm to the child, consequences and belief in a better way to get cooperation.

\section{Conclusion}

Parents' acceptance of pharmacological and aggressive behavioral management techniques is low [3]. However, their attitudes may change over time [10]. Therefore, parental awareness and acceptance of behavioral management techniques seems to be necessary [11]. In this study,

Table 1. The mean acceptance scores before and after intervention

\begin{tabular}{cccc}
\hline $\begin{array}{c}\text { Behavior Management } \\
\text { Techniques }\end{array}$ & Pre-test & Mean & P \\
\cline { 2 - 4 } & 28.47 & 59.62 & 0.000 \\
\hline Parental separation & 59.35 & 56.50 & 0.000 \\
\hline Voice control & 30.33 & 02.46 & 0.000 \\
\hline Active physical restraint & 41.32 & 19.45 & 0.000 \\
\hline General anesthesia & 92.13 & 53.27 & 0.000 \\
\hline Passive physical restraint & 38.12 & 28.20 & 0.000 \\
\hline Hand-over-mouth & & & \\
\hline
\end{tabular}


Table 2. Comparing the effect of informing methods on parental acceptance of pediatric behavior management techniques

\begin{tabular}{|c|c|c|c|}
\hline Behavior Management Techniques & Informing Methods & Mean Difference & $\mathbf{P}$ \\
\hline & Written/visual & -13.24 & 0.019 \\
\hline \multirow[t]{3}{*}{ General anesthesia } & Oral/visual & -14.53 & 0.008 \\
\hline & Written/oral & -1.29 & 0.990 \\
\hline & Written/visual & -1.20 & 0.993 \\
\hline \multirow[t]{3}{*}{ Voice control } & Oral/visual & -0.83 & 0.998 \\
\hline & Written/oral & 0.03 & 0.969 \\
\hline & Written/visual & -0.09 & 0.000 .1 \\
\hline \multirow[t]{3}{*}{ Parental separation } & Oral/visual & -2.12 & 0.978 \\
\hline & Written/oral & 2.22 & 0.976 \\
\hline & Written/visual & 2.50 & 0.854 \\
\hline \multirow[t]{3}{*}{ Hand-over-mouth } & Oral/visual & 5.46 & 0.314 \\
\hline & Written/oral & -7.96 & 0.068 \\
\hline & Written/visual & 15.64 & 0.001 \\
\hline \multirow[t]{3}{*}{ Active physical restraint } & Oral/visual & -19.44 & 0.000 \\
\hline & Written/oral & 3.79 & 0.749 \\
\hline & Written/visual & -7.22 & 0.298 \\
\hline \multirow[t]{2}{*}{ Passive physical restraint } & Oral/visual & 0.55 & 0.999 \\
\hline & Written/oral & 6.66 & 0.367 \\
\hline
\end{tabular}

the highest parental acceptance was related to the parental separation technique and the lowest acceptance was related to the hand-over-mouth and passive physical restraint techniques, which is in agreement with the results of other studies $[1,12-14]$. The hand-over-mouth technique is no longer recommended by the AAPD $[15,16]$. The use of papoose boards can lead to physical and psychological damage [5].

The hand-over-mouth and passive physical restraint techniques should be discarded. In this study, in comparison with the studies by Paryab et al. [3] and Razavi and Purtaji [17], the acceptance rate of general anesthesia had increased trend. The main reason for parents not accepting behavioral management techniques was the concern about their emotional problems and harm to the child. By increasing the awareness of parents, their worries can be reduced because the parents who are less concerned about their children's behavioral management techniques, show higher rate of acceptance [14]. It was concluded that parental informing increases parental acceptance of pediatric behavior management techniques and should be considered by dentists.

\section{Ethical Considerations}

\section{Compliance with ethical guidelines}

This study obtained its ethical approval from the Research Ethics Committee of Arak University of Medical Sciences (Code: IR.ARAKMU.REC.1398.102).

\section{Funding}

The present paper was extracted from the MSc. thesis of the first author, Department of Pediatric Dentistry, Faculty of Dentistry, Arak University of Medical Sciences, Arak.

\section{Authors' contributions}

All authors met the writing standards based on the recommendations of the International Committee of Medical Journal Editors (ICMJE).

\section{Conflicts of interest}

The authors declared no conflict of interest. 


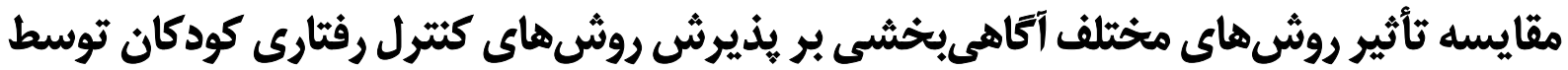

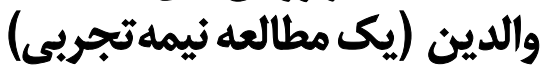

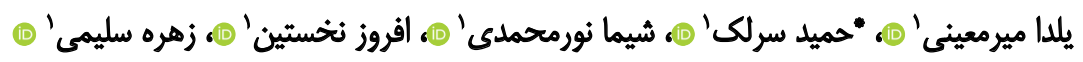 \\ 1. استاديار بخش دندانيزشكى كودكان، دانشكده دندانيزشكى، دانشعاه علوم يزشكى اراك، اراك، ايران.
}

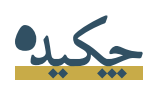

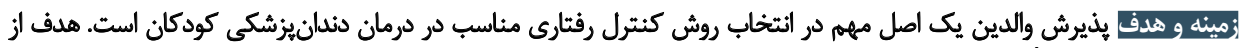

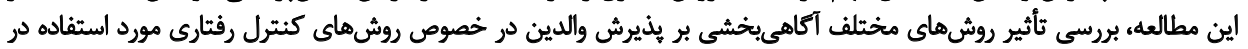

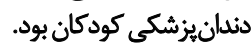

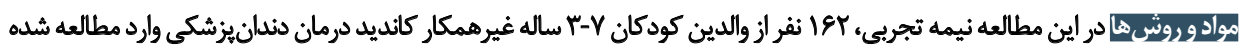

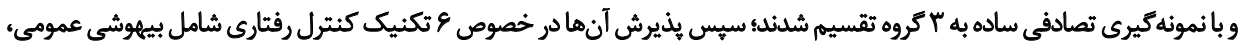

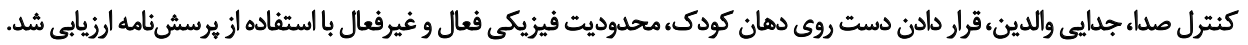

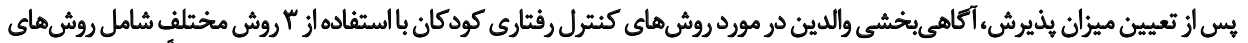

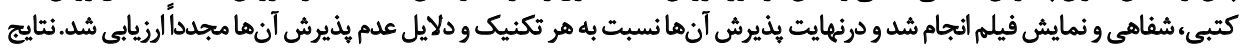

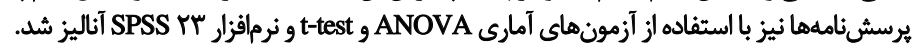

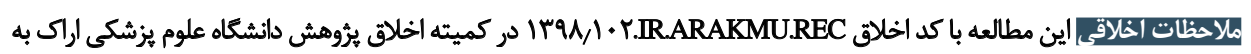
ثبتِ رسيده است.

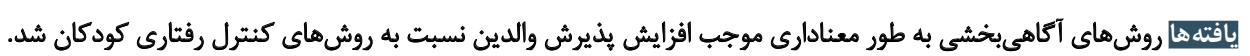

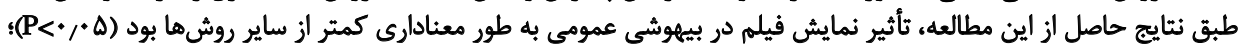

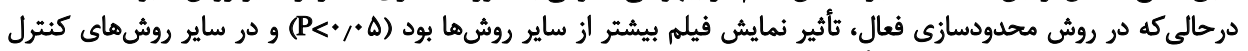

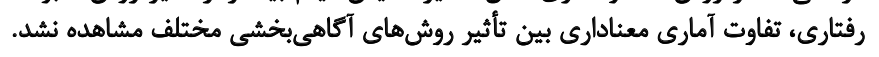

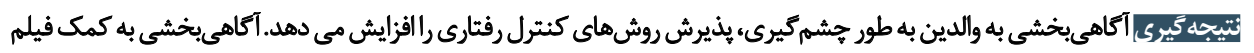

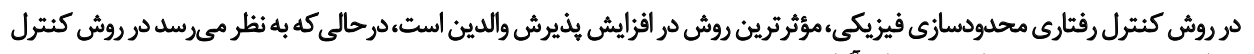

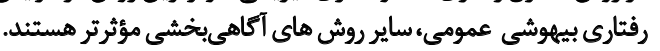

كه حتى در صورتى كه دندانيزشكان معتقد باشند اين روشها سائها

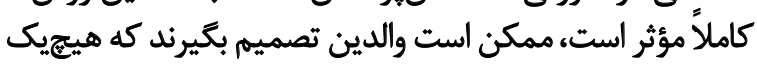

زمينه و هدف

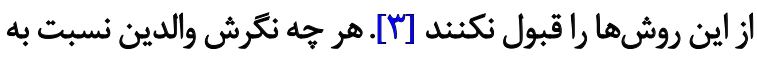

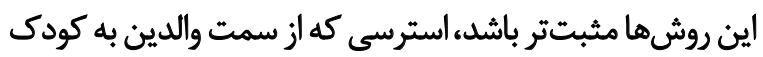

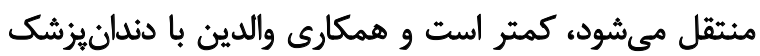

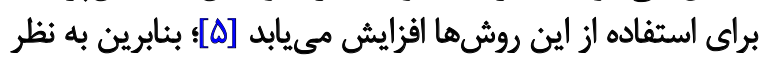

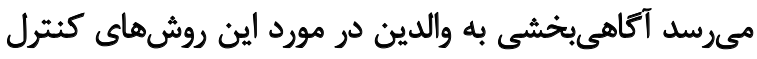

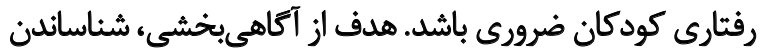

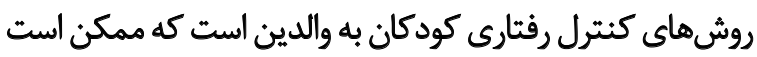

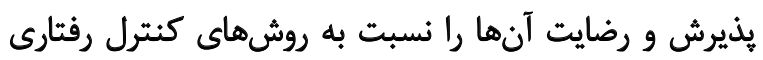

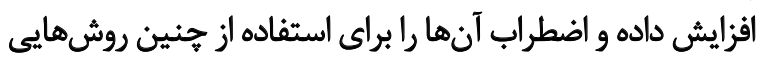

درصد بالايى از كودكان مراجعهكننده به دندانيزشكى هوديى

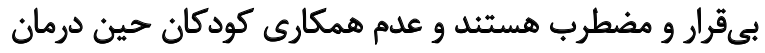

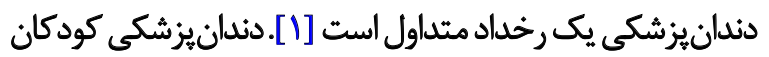

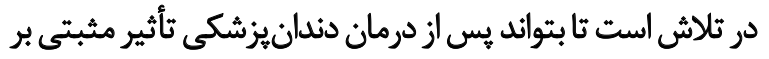

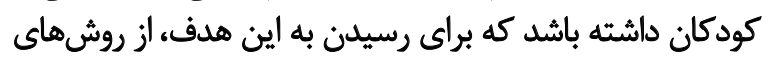

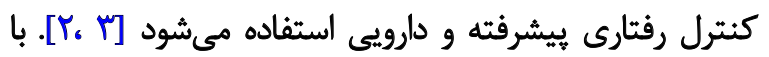

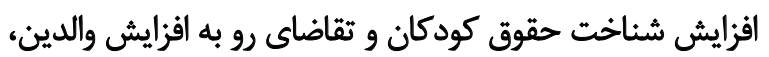

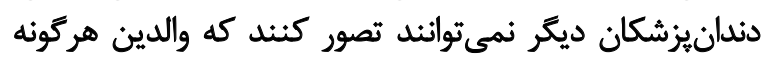

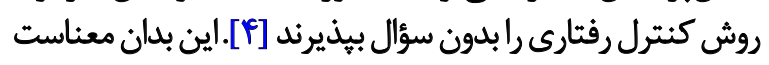

\section{:ويسنده مسئول:}

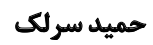

نشاني: اراك، دانشعاه علوم يزشكى اراك، دانشكده دنداثيزشكى، بخش دنداثيزشكى كودكان.

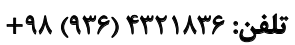
يست الكترونيكى: Dr.Hamidsarlak@yahoo.com 
اوردانسى و فورى دندانيزشكى دركودى؛ 9) والدين كودكانى كه

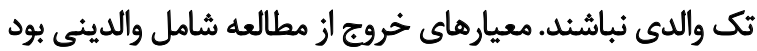

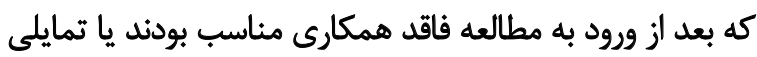

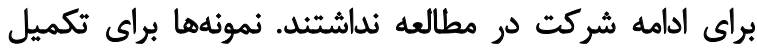

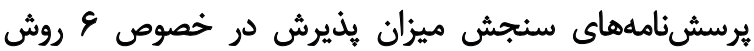

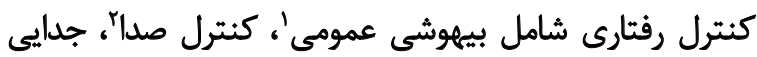

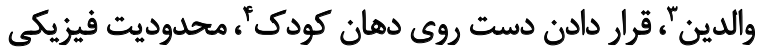
فعال هو غيرفعال ع به صورت تصادفى به سه كروه تقسيم شدند 1) كروه اول (آكاهىبخشى به صورت شفاهى): در اين كروه

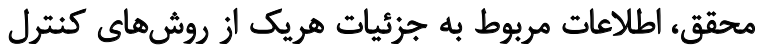
رفتارى كودكان به صورت شفاهى را به نمونهها ارائه كرد. (ب) كروه دوم (آكاهى بخشى به صورت كتبى): در اين كروها.

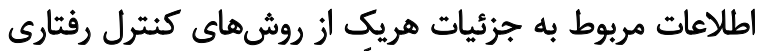

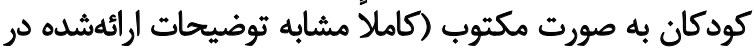

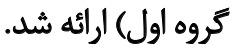

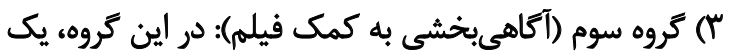

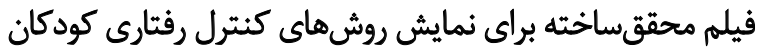
تهيه شد كه بدين منظور ه هوش

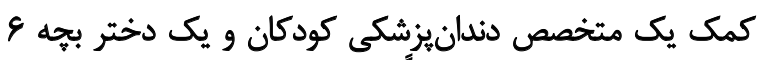

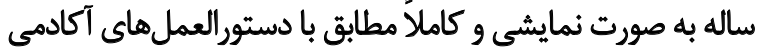

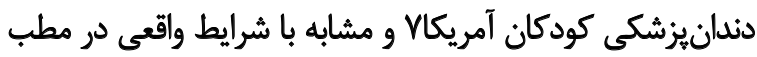

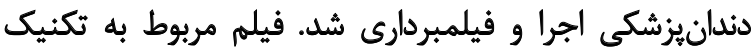

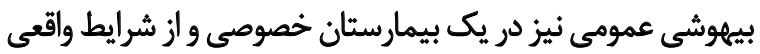

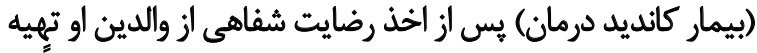

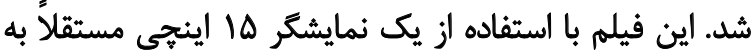

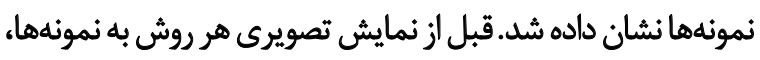

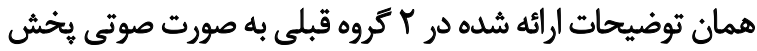

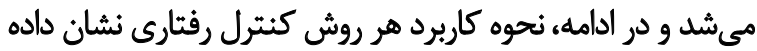

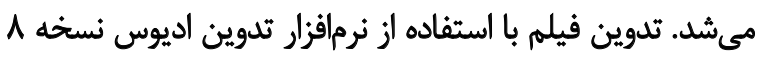
انجام شد و مدت زمان نمايش فيلم 1 دقيقه و ولا ثانيه بود.

اتر والدين در فهم مطالب و توضيحات دادهده دجار مشكلٍ

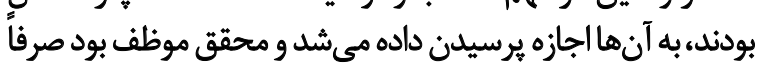

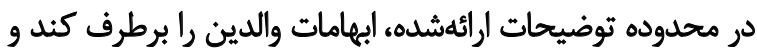

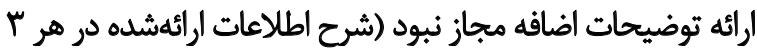

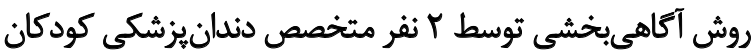
طبق رفرنس هاى دندانيزشكى كودكان و با زبانى ساده و قابل فيهمر
كاهش دهد [ب]]. روشهاى مختلفى براى ارائه اين اطلاعات به

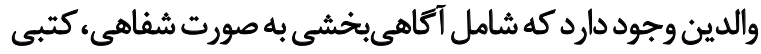

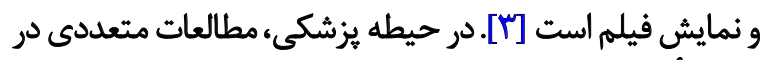

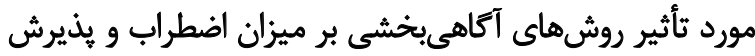

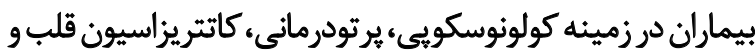
روماتولورى انجام شده است [؟]. اخيراً در دندانيزشكى كودكان نيز ميزان بذيرش روشام روشهاي

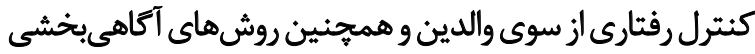

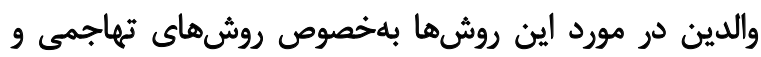

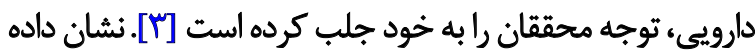

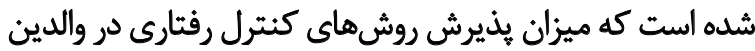

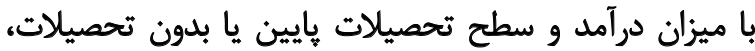

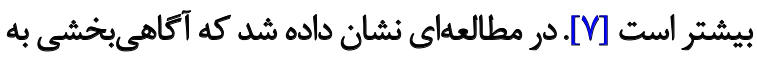

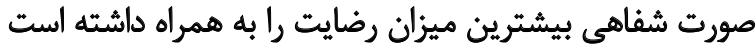

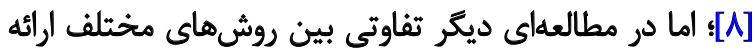

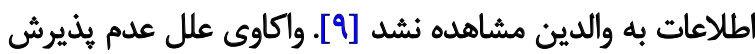

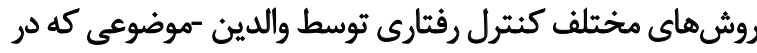

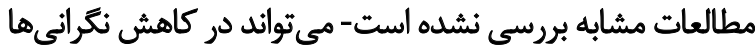

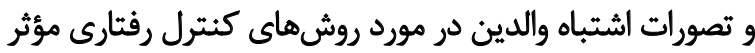

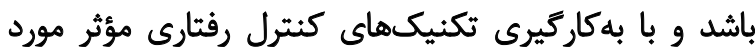

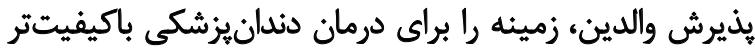

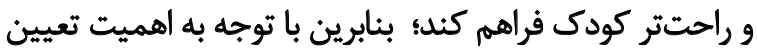

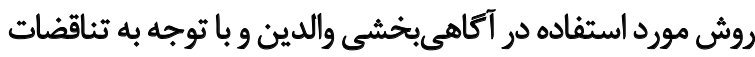

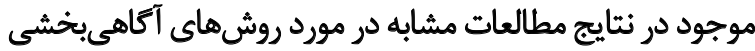

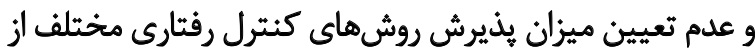

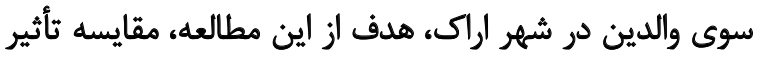

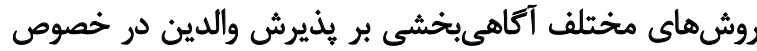

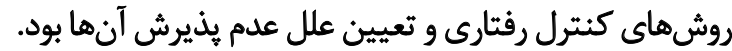

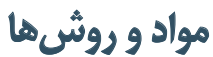

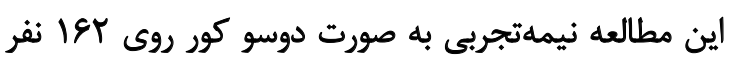

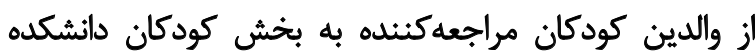

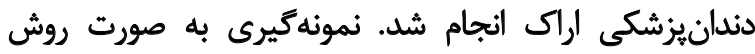

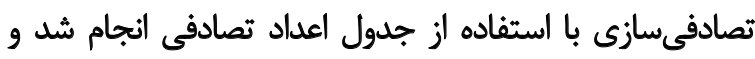

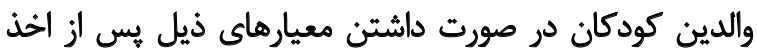
رضايتنامه آكاهانه و كتبى وارد مطالعه شدند (معيارهاى ورودي):

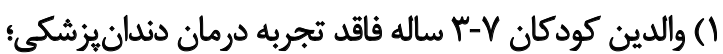

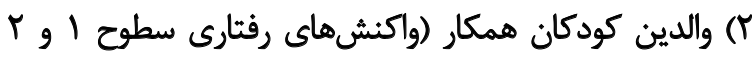

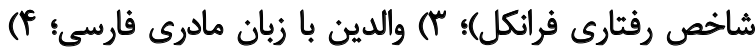

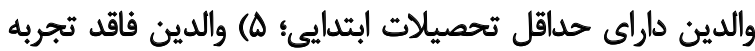

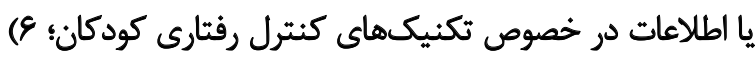

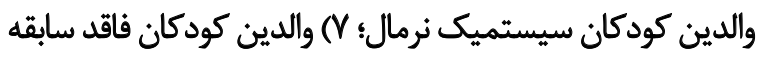

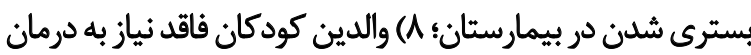


در جدول شماره ا آمده است. ميزان يذيرش روشئهاي كنترل

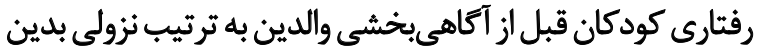

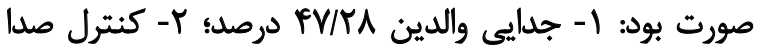

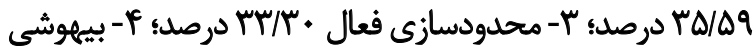

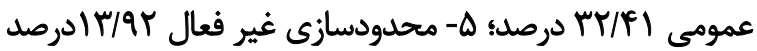

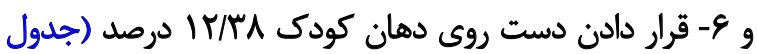

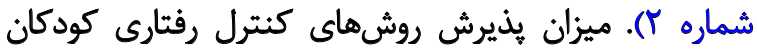

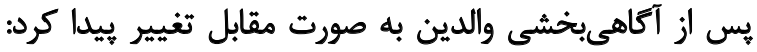

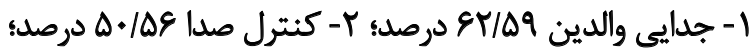

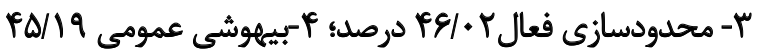

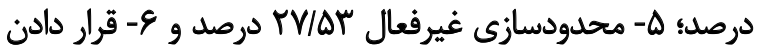

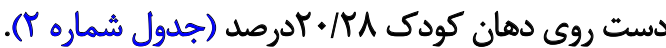
با توجه به جدول شماره rا، اثربخشى تمام روشهاى

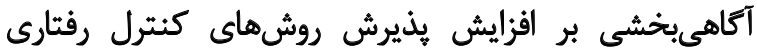

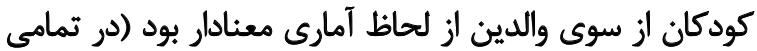

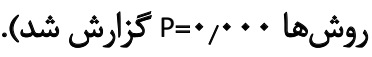

با توجه به نتايج جدول شماره سا، در تكنيك بيهوشى عمومى،

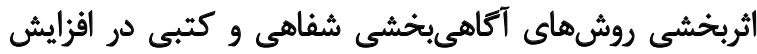

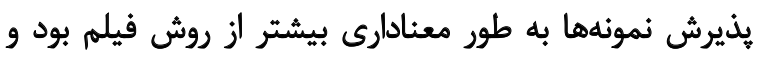

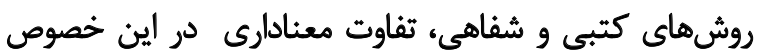

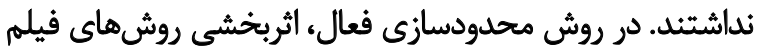

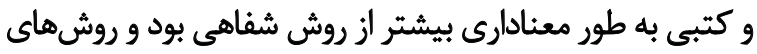

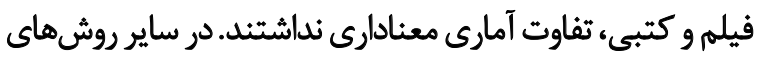

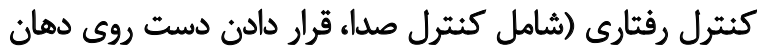

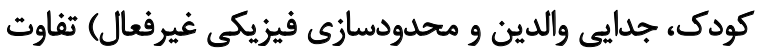

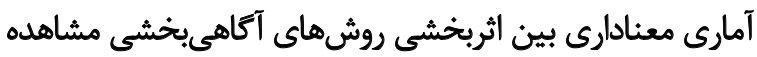
نشد و اثربخشى هر سه روش آكاهى بخشيى مرشى مشابه بود.

با توجه به نتايج جدول شماره f أ بدون تفكيك روشهاى

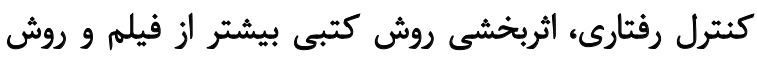

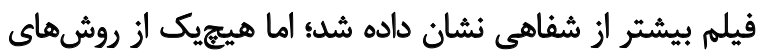
آكاهى بخشى از لحاظ آمارى تفاوت معنادارى نداشتئد. بيشترين علل عدم بذيرش والدين در خصوص روشهاي كنترل رفتارى شامل موارد زير بود:

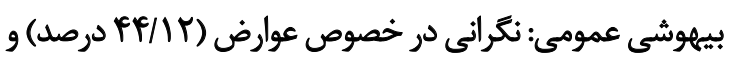
نكراني در مورد آسيب به كودى (FT/\&D

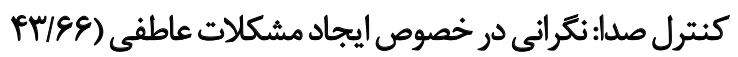

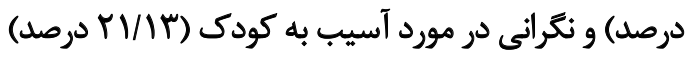
جدايى والدين: عدم تأثير مثبث اين روش در جلب همكارى

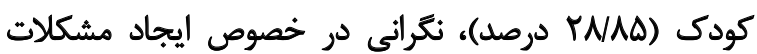

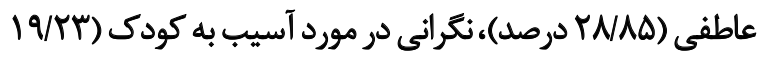

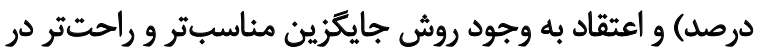

براى افراد غيردندانيزشك تدوين شد؛ يرسش نامهها يبيوست شده

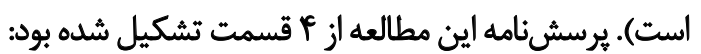
بخش اول: يرسشنامه دموكرافيك شامل سن و جنس والدين،

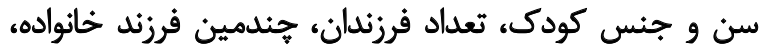

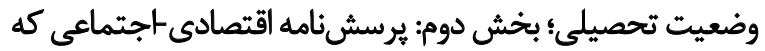

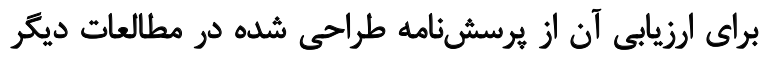

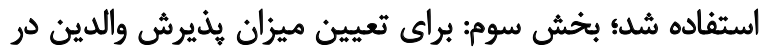

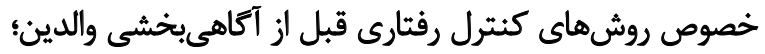

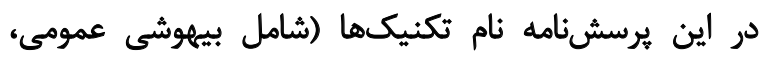

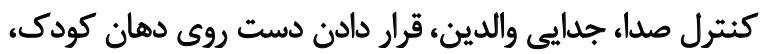

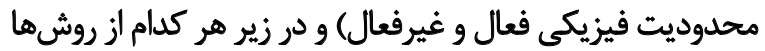

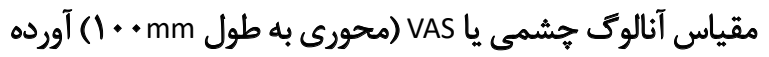

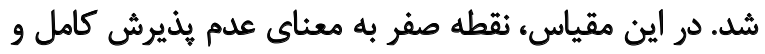

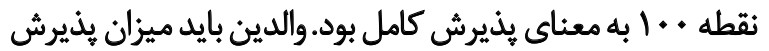

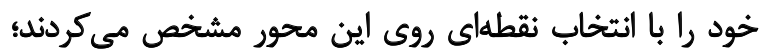

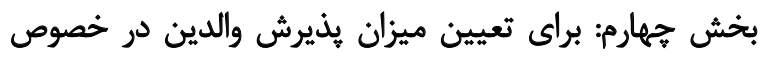

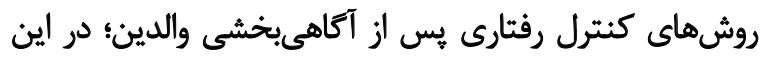

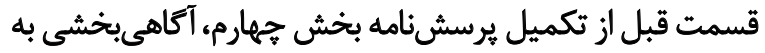

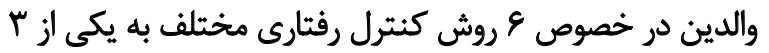

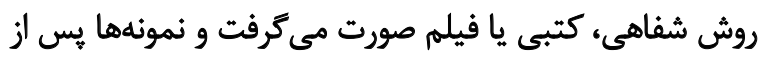

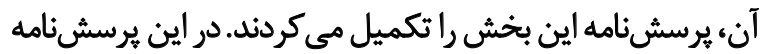

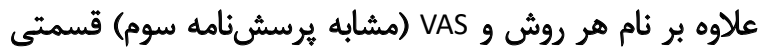

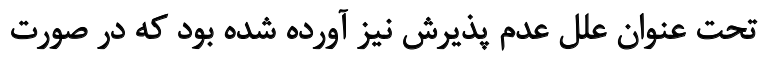

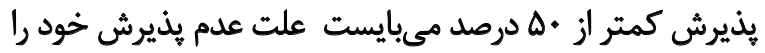

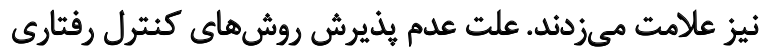

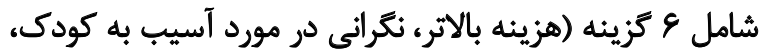

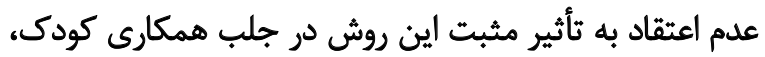

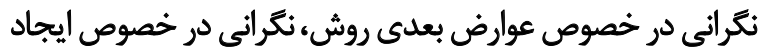

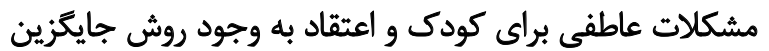
مناسبتر و راحتثر در جلب همكارى) بود. براى تحليل اطلاعات از شاخصهاى توصيفى شامل ميانكين،

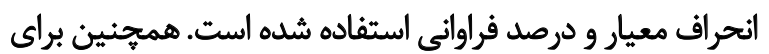

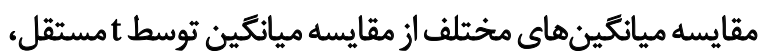

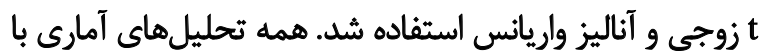

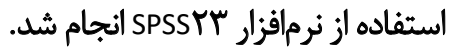

يافتهها

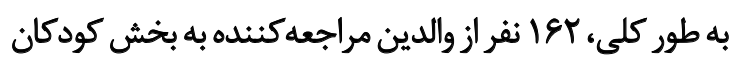

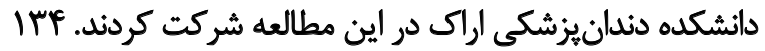

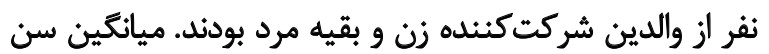

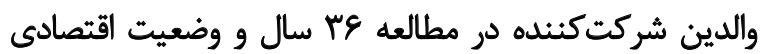

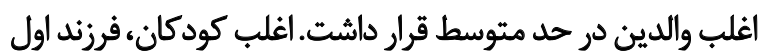

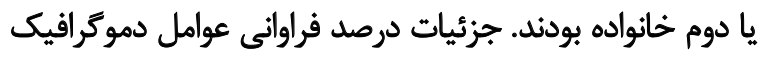


جدول ا. درصد فراوائى اطلاعات دموكرافيك مراجعهكنده

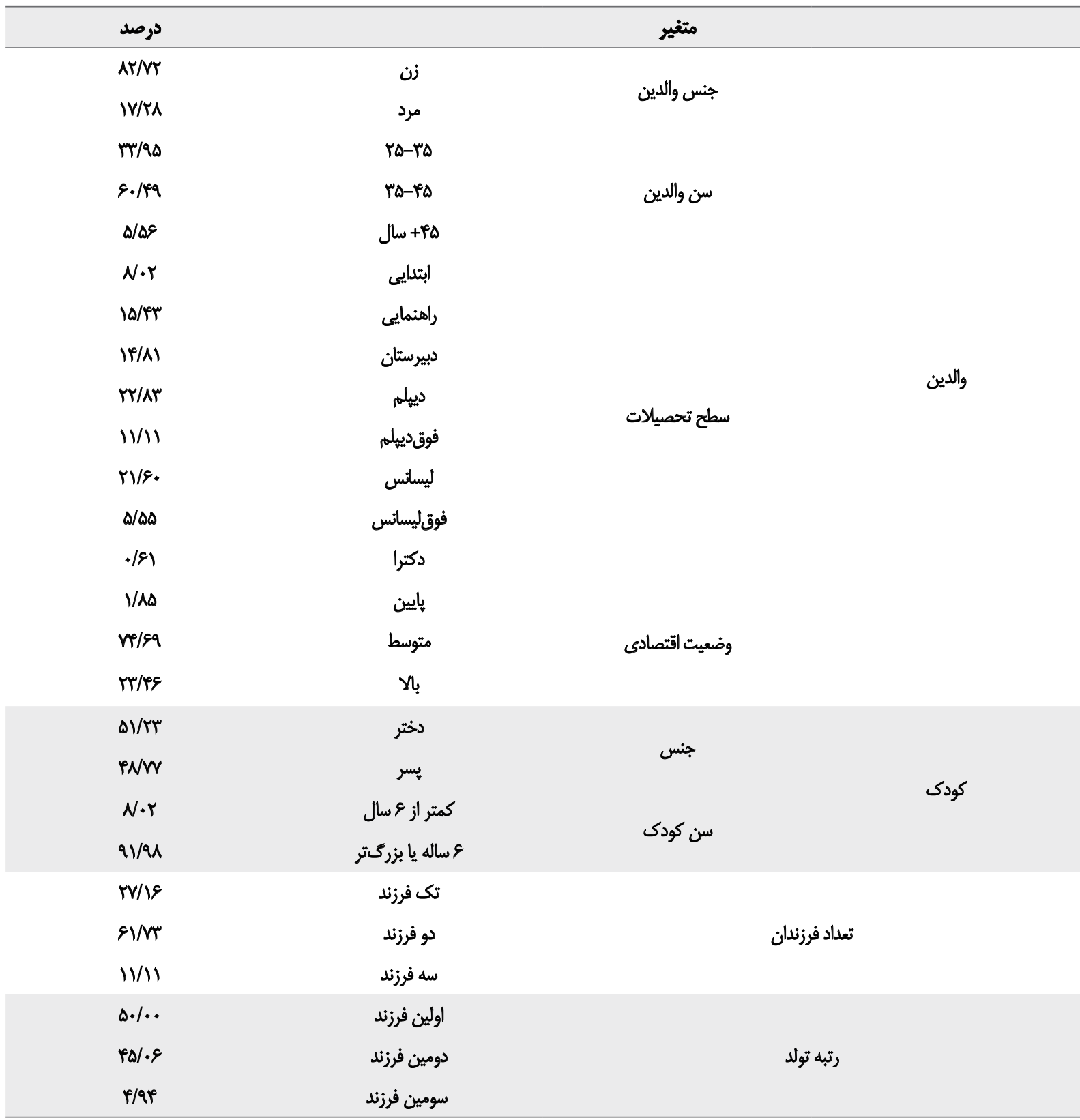

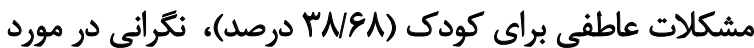

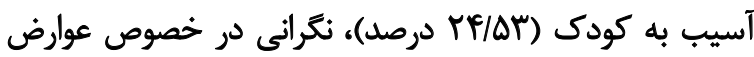

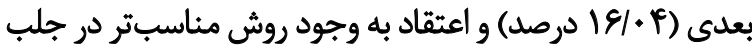

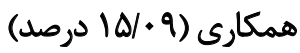

بحث

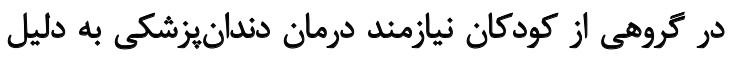

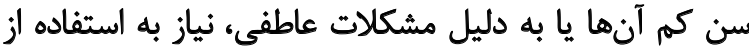
روشهاى كنترل رفتارى دارويى و تهاجمى افزايش بيدا نيدا مى كند.

$$
\text { جلب همكارى (\&\&/ آ درصد) }
$$

قرار دادن دست روى دهان كودكى نترانى در خصوص ايجاد

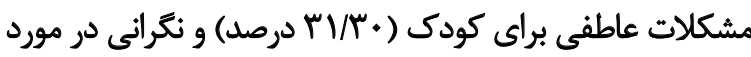

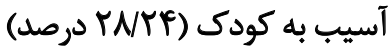
محدودسازى فيزيكى فعال: نكرانى در خصوص ايجاد مشكلات

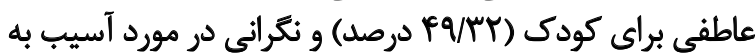

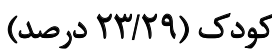
محدودسازى فيزيكى غيرفعال: نكرانى در خصوص ايجاد 
جدول T. مقايسه يذيرش والدين نسبت به روشهاى كنترل رفتارى كودكان قبل و بعد از آكاهىبخىى

\begin{tabular}{|c|c|c|c|}
\hline 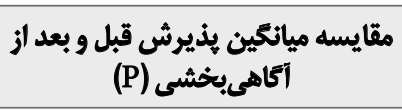 & ميانكين بذيرش بـ أكى از & ميانكين بذيخش قبل أكى از & روش كنترل رفتارى \\
\hline $.1 \ldots$ & gr/49 & PV/TA & جايى والدين \\
\hline$\%$ & $\Delta \cdot 1 \Delta \%$ & $r \omega / \Delta q$ & كتترل صدا \\
\hline$\%$ & Pel.r & $\Pi \pi / r^{\circ}$ & محدودسازي فعال \\
\hline.$\cdots$ & $P \Delta / 19$ & $\pi / P$ & بيهوشى عمومى \\
\hline$\%$ & rv/or & Ir/ar & محدودسازى غير فعال \\
\hline$\%$ & T./MA & $\mid r / \Psi A$ & قرار دادن دست روى دهان كودى \\
\hline
\end{tabular}

جدول ؟. مقايسه اثربخشى روشهاى آكاهي بخشى مختلف بر افزايش يذيرش والدين به تفكيك روشهاى كنترل رفتارى

\begin{tabular}{|c|c|c|c|}
\hline$P$ & تفاوت ميانكين & روشهاى مقايسهشده & روش كنترل رفتارى \\
\hline .1 .19 & $-I r / \pi r$ & فيلم-كبيى & \\
\hline.++1 & $-\mid \varphi / \Delta r$ & فيلم-شفاهى & بيهوشى عمومى \\
\hline.$/ 99$. & $-1 / 49$ & كتبى-شفاهى & \\
\hline ./994 & $-1 / 4$ & كتبى-شفاهى & \\
\hline.$/ 94$ & - & شفاهى-فيلم & كتترل صدا \\
\hline$. / 8<9$ & $r / \%$ & فيلم-كبيى & \\
\hline $1 / \ldots$ & -.1 .9 & كتبى-شفاهى & \\
\hline.$/ 9 \vee \wedge$ & $-t / r$ & شفاهى -فيلم & جدايى والدين \\
\hline.$/ 9 \vee 9$ & $r / T r$ & فيلم-كبيى & \\
\hline . IASF & $r / \Delta$. & كتيى -شفاهى & \\
\hline.$/ M I F$ & $\Delta / \varphi$ & شفاهى -فيلم & قرار دادن دست روى دهان كودى \\
\hline .1 .81 & $-V / q \varepsilon$ & فيلم-كبي & \\
\hline.$/ . .1$ & $\mid \omega / 9 r$ & كتبي -شفاهى & \\
\hline.$\%$ & $-19 / 94$ & شفاهى -فيلم & محدودسازى فعال \\
\hline.$/ M e q$ & $r / v q$ & فيلم-كبيى & \\
\hline . /ra & $-V / T r$ & كتبى-شفاهى & \\
\hline.$/ 999$ &.$/ \Delta \Delta$ & شفاهى-فيلم & محلودسازى غيرفعال \\
\hline.$/ M g V$ & s/\$8 & فيلم-كتيى & \\
\hline
\end{tabular}

كاهش همكارى آنها شود [11].

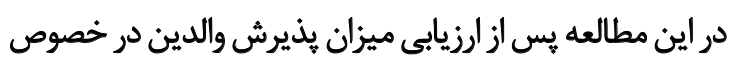

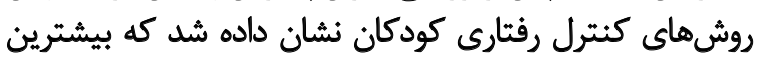

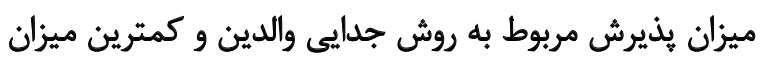

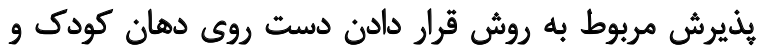

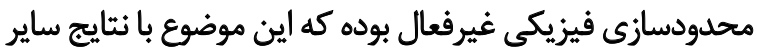

در بسيارى از مطالعات انجام شده، ميزان بذيرش والدين در

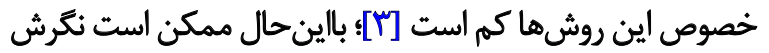

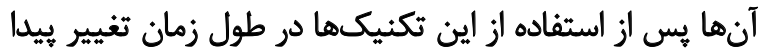

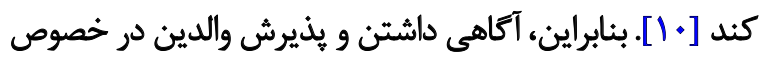

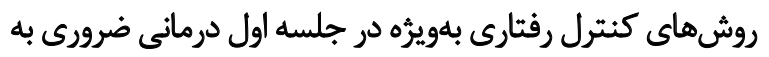

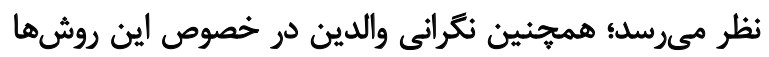

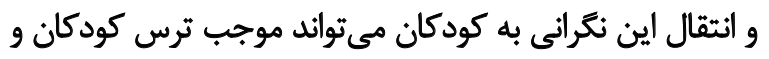


جدول F. مقايسه اثربخشى روش هاى آكاهى بخشى مختلف بر يذيرش روش هاى كنترل رفتارى(بدون تفكيك روش هاى كنترل رفتارى)

\begin{tabular}{|c|c|c|}
\hline $\mathbf{P}$ & تفاوت ميانكين & روشهاى آكاهى بخشى مقايسهشده \\
\hline.$/ 1 \Delta A$ & V/TMA & كتبى -شفاهى \\
\hline ./9rV & $1 / A \cdot r A$ & كتبى-فيلم \\
\hline +/944 &.$- / \% \cdot N A F$ & شقاهى-خيلم \\
\hline
\end{tabular}

كنترل رفتارى (شامل روش كتبى، روش نوشتارى و نمايش فيلم)

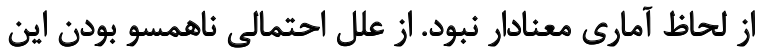

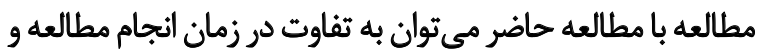

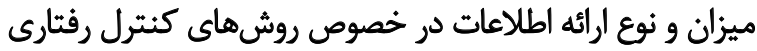

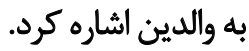

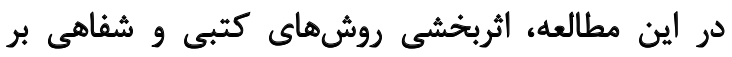

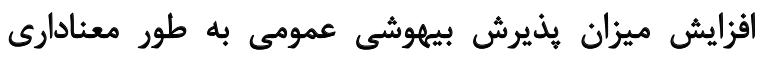

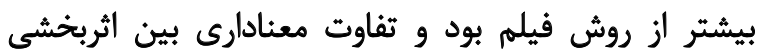

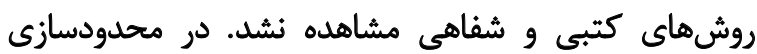

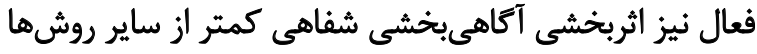

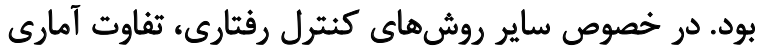

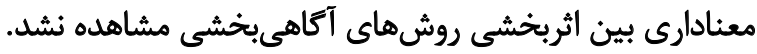

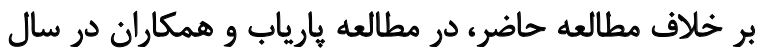

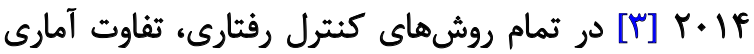

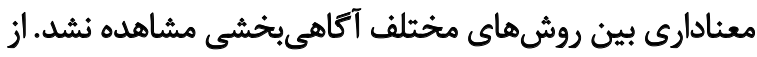

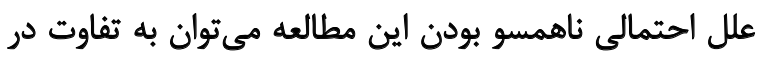

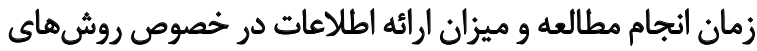
كنترل رفتارى به والدين اشاره كرد.

هيج مطالعهاى تاكنون علل عدم يذيرش روش إهاى كنترل

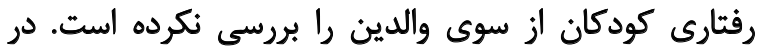

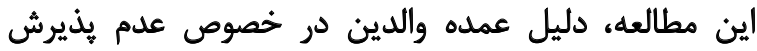

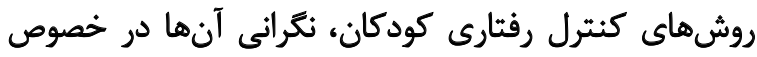

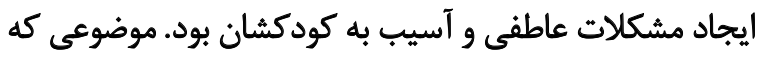

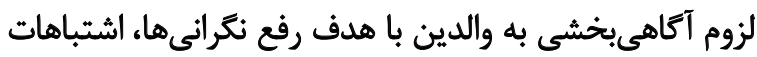

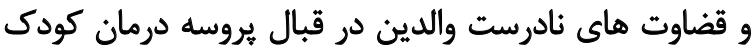

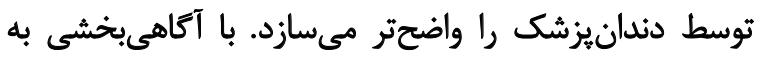

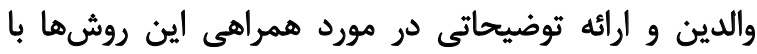

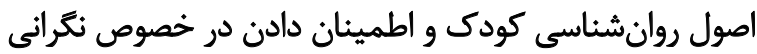

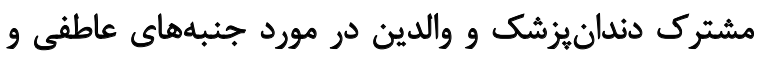

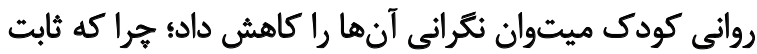

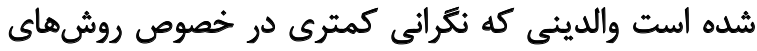

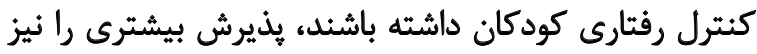

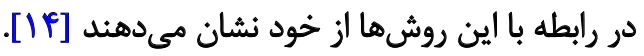

مطالعات همسو است [ [ | - | | ، ]. در اين مطالعه، كمترين ميزان

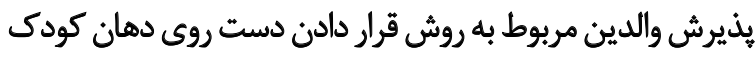

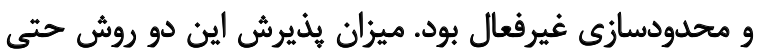

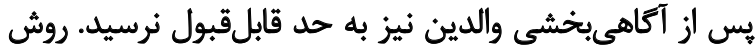

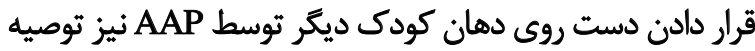

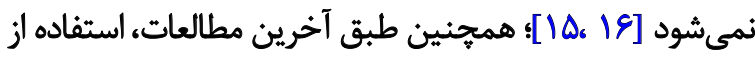

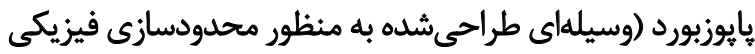

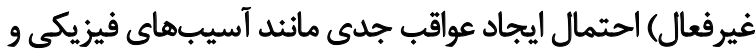

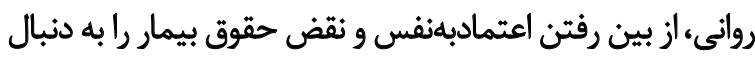

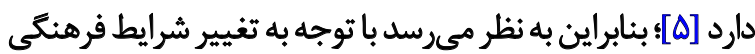

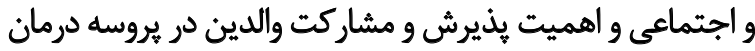

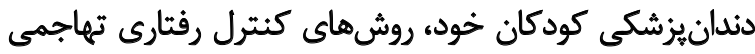

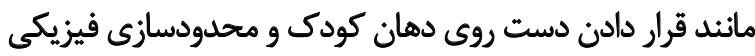

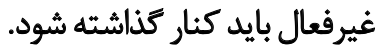

در اين مطالعه در مقايسه با مطالعه بارياب و همكاران در سال

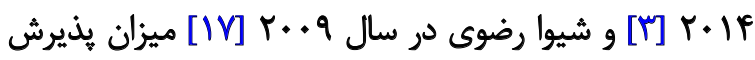

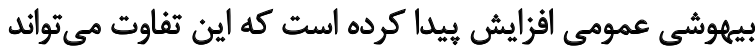

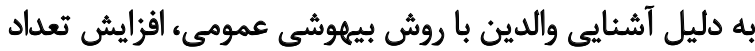

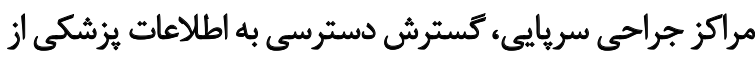

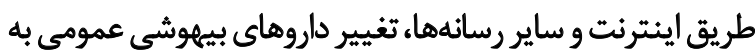

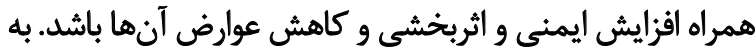

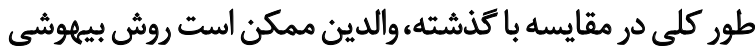

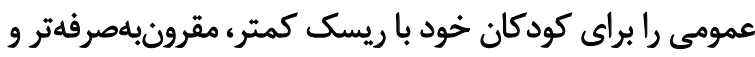

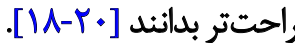

با توجه به نتايج اين مطالعه، اثربخشى تمام روشهاي

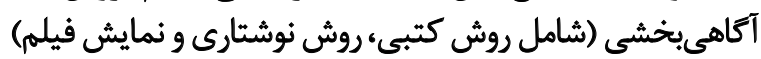

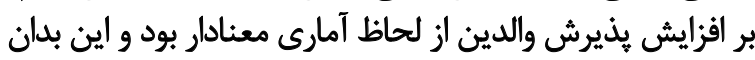

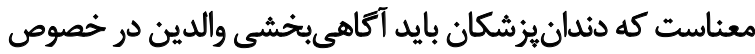

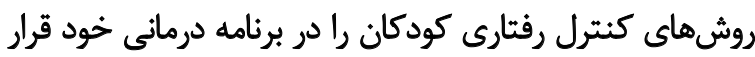

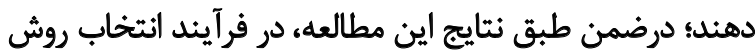

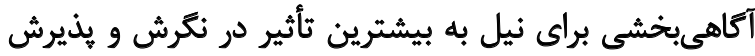

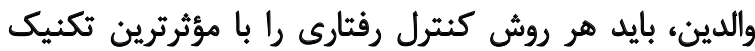

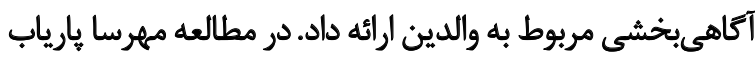

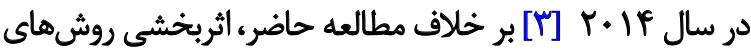


تمامى نويسندكان معيارهاى استاندارد نويسندكى بر اساس

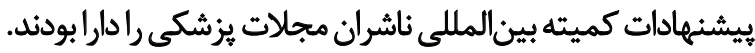

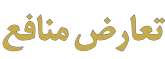

بدينوسيله نويسندكان تصريح مى كنئد كه هيجزونه تضاد مثافعى در خصوص ثرؤهش حاضر وجود ندارد.
در جامعه مورد بررسى ما، ترثيب بذيرش و

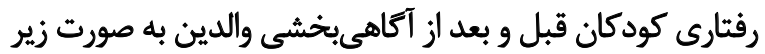

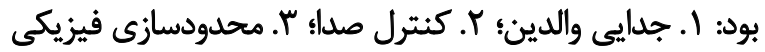

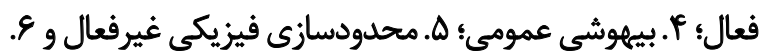

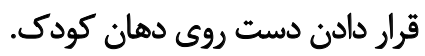

طبق نتايج اين مطالعه، آكاهىبخشى والدين موجب افزايش رئ رئري

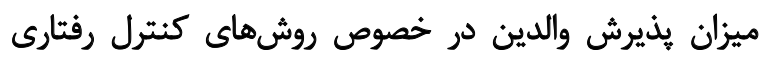

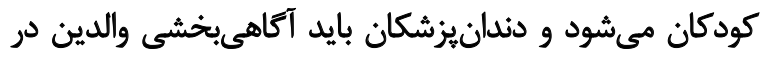

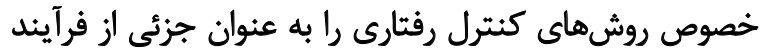

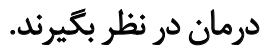

دليل عمده والدين در خصوص عدم يذيرش روش روشهاي كنترل

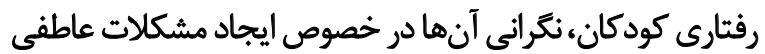

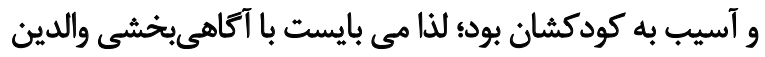

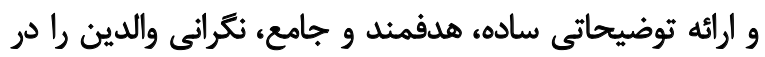
خصوص اثرات منفى و عوارض اين روشهادى كنترل رفائ رفتارى

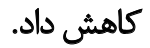

$$
\text { بيشنهانهان }
$$

(1) ييشنهاد مي شود با توجه به تأثير تجربه قبلى دندانيز ئشكى

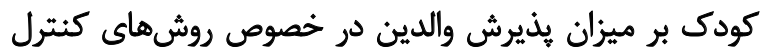
رفتارى كودكان، اين متغير نيز در مطالعات آينده بروسى شوش شود.

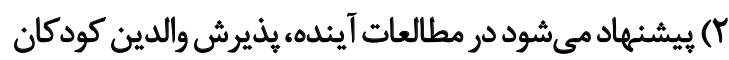

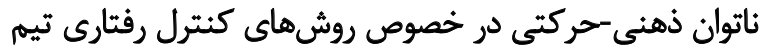
دندانيزشكى ارزيابي شود.

$$
\text { محدوديتها }
$$

محدوديت اصلى مطالعه حاضر، تهيه فيلم مناسب، تويا و مطابق با اصول تأكيدشده AAPD در مقوله كنترل رفتارى بود.

مالاحظات اخلاقى

\section{ييروى أز أصول انخلاق يؤوهش}

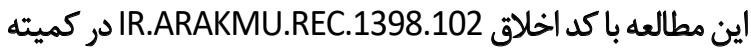

$$
\begin{aligned}
& \text { اخلاق يُروهش دانشكاه علوم بزشكى اراك به ثبت رسيده است. }
\end{aligned}
$$

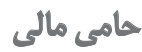

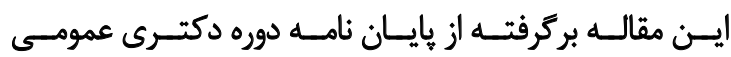

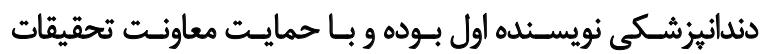

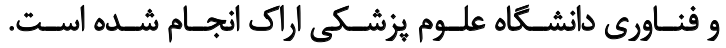




\section{References}

[1] Jafarzadeh M, Kooshki F, Malekafzali B, Ahmadi S. Attitude of parents referred to the department of pediatric dentistry towards different behavioral management techniques used in pediatric dentistry. J Dent Sch Shahid Beheshti Univ Med Sci. 2015; 33(1):44-50.

[2] Boka V, Arapostathis K, Vretos N, Kotsanos N. Parental acceptance of behaviour-management techniques used in paediatric dentistry and its relation to parental dental anxiety and experience. Eur Arch Paediatr Dent. 2014; 15(5):333-9. [DOI:10.1007/s40368-014-0119-y] [PMID]

[3] Paryab M, Afshar H, Mohammadi R. Informing parents about the pharmacological and invasive behavior management techniques used in pediatric dentistry. J Dent Res Dent Clin Dent Prospects. 2014;8(2):95100. [DOI:10.5681/joddd.2014.017] [PMID] [PMCID]

[4] Elango I, K Baweja D, K Shivaprakash P. Parental acceptance of pediatric behavior management techniques: A comparative study. J Indian Soc Pedod Prev Dent. 2012; 195-200. [DOI:10.4103/0970-4388.105010] [PMID]

[5] McDonald RE, Avery DR. Foreword. In: Dean JA, editor. McDonald and Avery's Dentistry for the Child and Adolescent (Tenth Edition). St. Louis: Mosby; 2016. [DOI:10.1016/B978-0-323-28745-6.06001-4]

[6] Adewumi A HM, king JM. Children and informed consent: A study of children's perceptions and involvement in consent to dental treatment. Br Dent J 2001; 191:256-9. [DOI:10.1038/sj.bdj.4801157a] [PMID]

[7] Abushal MS, Adenubi JO. Attitudes of Saudi parents toward behavior management techniques in pediatric dentistry. J Dent Child (Chic). 2003; 70(2):104-10. [PMID]

[8] Wright GZKA, Wright GZ. Behavior management in dentistry for children. Hoboken; John Wiley \& Sons, Inc: 2014. [DOI:10.1002/9781118852446]

[9] Eaton JJ, McTigue DJ, Fields HW, Jr., Beck M. Attitudes of contemporary parents toward behavior management techniques used in pediatric dentistry. Pediatr Dent. 2005; 27(2):107-13. [PMID]

[10] Tsuchihashi N UN, Takagi Y, Miwa Z, Sugimo K. Internal stress in children and parental attitude to dental treatment with passive restraint. Pediatr Dent J 2012; 22:170-7. [DOI:10.1016/S0917-2394(12)70268-9]

[11] Eshghi A, Rezaeifar M, jafarzadeh Samani M, Malekafzali B, Eftekhari M. Evaluation of parental view toward dental treatment under general anesthesia in Isfahan. J Adv Med Biomed Res. 2010;18(73):67-75. http://zums.ac.ir/journal/article-1-1304-fa.html

[12] Al Daghamin S BM AS, AlObaidi F, Kakti A. Behavior management techniques in pediatric dentistry: how well are they accepted? Acad J Ped Neonatol. 2017; 5:1-6.

[13] Acharya S, Acharya Sonu S, Leite Cavalcanti A, Wilney W, Padilha N. parental acceptance of various behaviour management techniques used in pediatric dentistry: a pilot study in odisha, india. Pesquisa Brasileira em Odontopediatria e Clínica Integrada. 2017; 2017(1):e3728. [DOI:10.4034/PBOCI.2017.171.26]

[14] Allen KD, Hodges ED, Knudsen SK. Comparing four methods to inform parents about child behavior management: how to inform for consent. Pediatr Dent. 1995; 17(3):180-6. [PMID]

[15] Roberts JF, Curzon ME, Koch G, Martens LC. Review: Behaviour management techniques in paediatric dentistry. Eur Arch Paediatr Dent. 2010; 11(4):166-74. [DOI:10.1007/BF03262738] [PMID]

[16] American Academy of Pediatric Dentistry (AAPD). behavior guidance for the pediatric dental patient. Am Acad Pediatr Dent. https://www. aapd.org/globalassets/media/policies_guidelines/bp_behavguide.pdf
[17] Razavi S, Purtaji B. Determining the behavior management technique's acceptance of mothers referred to the department of pediatric dentistry in Qazvin (2007). J Qazvin Univ Med Sci. 2009; 13(3):81-6. http://journal. qums.ac.ir/article-1-879-en.htm

[18] American Academy of Pediatric Dentistry. Guideline on behavior guidance for the pediatric dental patient. 2009; 30(7):125-33.

[19] Luis de León J1 GJF, Bellet Dalmau U. Acceptance by Spanish parent of behaviour management techniques used in paediatric dentistry. Eur Arch Paediatr Dent. 2010; 11(4):175-8. [DOI:10.1007/BF03262739] [PMID]

[20] Saleh M MS, Sabiha A. Parental attitude toward behavioral management techniques in dental practice with school children in Kuwait. Med Princ Pract. 2011; 20:350-55. [DOI:10.1159/000323758] [PMID] 
This Page Intentionally Left Blank 